\title{
Myocardial T1 and Extracellular Volume Fraction Mapping at 3 Tesla
}

\author{
Jason J Lee ${ }^{1}$, Songtao Liư ${ }^{1,2}$, Marcelo S Nacif', Martin Ugander ${ }^{3}$, Jing Han ${ }^{4}$, Nadine Kawel ${ }^{1}$, Christopher T Sibley ${ }^{1,2}$, \\ Peter Kellman ${ }^{3}$, Andrew E Arai ${ }^{3}$ and David A Bluemke ${ }^{1,2^{*}}$
}

\begin{abstract}
Background: To compare 11 heartbeat (HB) and $17 \mathrm{HB}$ modified lock locker inversion recovery (MOLLI) pulse sequence at $3 \mathrm{~T}$ and to establish preliminary reference values for myocardial $\mathrm{T} 1$ and the extracellular volume fraction (ECV).

Methods: Both phantoms and normal volunteers were scanned at $3 \mathrm{~T}$ using $11 \mathrm{HB}$ and $17 \mathrm{HB}$ MOLLI sequence with the following parameters: spatial resolution $=1.75 \times 1.75 \times 10 \mathrm{~mm}$ on a $256 \times 180$ matrix, TI initial $=110 \mathrm{~ms}$, $\mathrm{Tl}$ increment $=80 \mathrm{~ms}$, flip angle $=35^{\circ}, \mathrm{TR} / \mathrm{TE}=1.9 / 1.0 \mathrm{~ms}$. All volunteers were administered Gadolinium-DTPA (Magnevist, $0.15 \mathrm{mmol} / \mathrm{kg}$ ), and multiple post-contrast MOLLI scans were performed at the same pre-contrast position from 3.5-23.5 minutes after a bolus contrast injection. Late gadolinium enhancement (LGE) images were also acquired 12-30 minutes after the gadolinium bolus.
\end{abstract}

Results: T1 values of $11 \mathrm{HB}$ and $17 \mathrm{HB}$ MOLLI displayed good agreement in both phantom and volunteers. The average pre-contrast myocardial and blood T1 was $1315 \pm 39 \mathrm{~ms}$ and $2020 \pm 129 \mathrm{~ms}$, respectively. ECV was stable between 8.5 to 23.5 minutes post contrast with an average of $26.7 \pm 1.0 \%$.

Conclusion: The $11 \mathrm{HB}$ MOLLI is a faster method for high-resolution myocardial T1 mapping at 3T. ECV fractions are stable over a wide time range after contrast administration.

\section{Background}

Cardiovascular magnetic resonance (CMR) is a noninvasive imaging method for accurate assessment of myocardial anatomy and function. A unique feature of CMR is the ability to use the proton relaxation times, such as $\mathrm{T} 1, \mathrm{~T} 2$, and $\mathrm{T} 2 *$, to characterize myocardial or vascular tissue [1]. These values are largely dependent on the physical and chemical environments of water protons in the tissue. Myocardial fibrosis is one of the most common histological features of the failing heart [2]. Late gadolinium enhancement (LGE) has been the standard of reference for detecting focal myocardial fibrosis in clinical practice [3]. LGE relies on the differences in signal intensity between scarred and adjacent normal myocardium to generate image contrast. Because this method lacks a normal myocardium reference value, LGE is unlikely to detect the presence of diffuse

\footnotetext{
* Correspondence: bluemked@nih.gov

'Radiology and Imaging Sciences, National Institutes of Health Clinical Center, Bethesda, MD, USA

Full list of author information is available at the end of the article
}

myocardial fibrosis tissue. T1 mapping, is a promising quantitative method for detecting diffuse myocardial fibrosis.

The Modified Look-Locker Inversion-recovery (MOLLI) technique uses electrocardiogram (ECG)-gated image acquisition at end-diastole and merges images from multiple consecutive inversion-recovery experiments into one data set. A high-resolution T1 map of the myocardium can be acquired in one breath hold $[4,5]$. The traditional MOLLI protocol uses three inversion-recovery blocks to acquire 11 images over 17 heartbeats (HB). These longer breath holds may limit its clinical applications in patients with impaired respiratory conditions due to cardiac diseases. Several new protocols have been proposed to reduce the MOLLI acquisition time [6,7].

Post contrast myocardial $\mathrm{T} 1$ values are affected by a variety of factors. These include, but are not limited to, the type and amount of gadolinium contrast injected, post contrast scan times, renal function, hematocrit, $B_{o}$ field, body composition, as well as pre-contrast T1 time. 
T1 mapping must take into account corrections for these variables before further analyses can be made [8] Normalizing myocardial T1 in relationship to blood T1 avoids much of these complexities. Extracellular volume fraction (ECV), fibrosis index [9], or volume of distribution $[10,11]$ are similar methods that measure the myocardial extracellular volume fraction by normalizing myocardial R1 change with blood R1 change and by correction for hematocrit. Although previous studies show that myocardial ECV quantifications correlate with diffuse myocardial fibrosis $[10,12]$, these values have not yet been reported for $3 \mathrm{~T}$ CMR.

In this study, we evaluated the accuracy of the $11 \mathrm{HB}$ and $17 \mathrm{HB}$ MOLLI sequences at 3T through phantom and normal volunteer studies. Preliminary reference values of both pre-contrast and post-contrast were quantified at different time points. Additionally, preliminary reference ECV values were also estimated from the normal volunteers.

\section{Methods}

All experiments were performed at a 3 Tesla scanner (Verio, Siemens Medical Systems, Erlangen, Germany) with a 32-channel cardiovascular array coil (Invivo, Orlando, FL).

\section{CMR parameters}

The 17 HB MOLLI consists of three inversion blocks: 3 images are acquired after each of the first two inversion pulses and 5 images are acquired after the third inversion pulse. The 11 HB MOLLI consists of 2 inversion blocks: 3 images are acquired after the first inversion pulse and 5 images are acquired after the second inversion pulse. Both the $17 \mathrm{HB}$ and $11 \mathrm{HB}$ protocol share all other parameters: 3 heartbeat pause between inversion blocks, nonsegmented steady state free precession readout, field of view: $360 \times 290 \mathrm{~mm}$, matrix: $256 \times 180$, slice thickness $10 \mathrm{~mm}$, TR/TE: $1.9 / 1.0 \mathrm{~ms}$, minimum inversion time 110 $\mathrm{ms}$, inversion time increment $80 \mathrm{~ms}$, flip angle 35 degrees, GRAPPA parallel imaging factor 2 .

\section{Phantom Studies}

The phantoms containing agarose gel doped with cupric sulfate were scanned using both $17 \mathrm{HB}$ and $11 \mathrm{HB}$ MOLLI sequence with simulated heart rates from 40 to 110 beats per minute (BPM) in increments of 10 heartbeats. To determine the reference $\mathrm{T} 1$ time of each phantom, standard inversion-recovery spin echo sequence with the same FOV and matrix size were acquired at 20 different TIs with a TR of $10 \mathrm{sec}$, and a TE of $8.5 \mathrm{~ms}$.

\section{Human Volunteer Studies}

Eleven healthy human subjects (six male and five female, ages $36 \pm 13$ years) without cardiovascular disease were enrolled for this study. All participants have normal ECG and were briefed on the procedure they were to undertake and all volunteers gave institutional review board approved written informed consent. $11 \mathrm{HB}$ and 17 HB MOLLI images were acquired at mid-ventricular short axis view. All subjects were administered Gadolinium-DTPA contrast (Magnevist, $0.15 \mathrm{mmol} / \mathrm{kg}$ ) at an injection rate of $2 \mathrm{ml} / \mathrm{s}$ followed by a $20 \mathrm{ml}$ saline flush. Multiple post-contrast MOLLI scans were performed at the same pre-contrast position from 3.5, 5, 8.5, 13.5, 18.5 and 23.5 minutes after contrast injection. Standard phase sensitive inversion recovery late gadolinium enhancement imaging was acquired at 12-30 minutes after contrast injection [13]. Blood samples were taken 1 to 4 hours prior to the CMR scan to determine the hematocrit and creatinine.

\section{Image Analysis}

For both phantom and human studies, T1 maps were calculated using MRmap [14] and stored in Digital Imaging and Communications in Medicine (DICOM) Format. To extract myocardial T1 values for the human subjects, endocardial and epicardial contours were manually traced using QMass MR 7.2 (Leiden, Netherlands). The blood pool regions were carefully excluded when the regions of interest were traced. Phantom MOLLI T1 values of different heart rates were fitted to IR-SE T1 values by $2^{\text {nd }}$ order polynomial function and pre-contrast in-vivo MOLLI T1 values were corrected according to the phantom result. ECV values were calculated using [11]:

$$
\begin{aligned}
& \Delta \mathrm{R} 1_{\text {myo }}=1 / \mathrm{T} 1_{\text {myo-post }}-1 / \mathrm{T} 1_{\text {myo-pre }} \\
& \Delta \mathrm{R} 1_{\text {blood }}=1 / \mathrm{T} 1_{\text {blood-post }}-1 / \mathrm{T} 1_{\text {blood }}-\text { pre } \\
& \mathrm{ECV}=\Delta \mathrm{R} 1_{\text {myo }} / \Delta \mathrm{R} 1_{\text {blood }} \times(100-\mathrm{HCT})
\end{aligned}
$$

where ECV and HCT are give as percentage.

\section{Data Analysis}

Statistical analyses were performed using SAS 9.1(Cary, North Carolina, USA) and Medcalc 11.6 (Medcalc Software, Belgium). Bland-Altman plots were used to describe the difference of $\mathrm{T} 1$ values between $17 \mathrm{HB}$ and $11 \mathrm{HB}$ protocols. To statistically compare the T1 values of two protocols, a general linear mixed model was used. The MOLLI protocol was included in the model as a fixed effect, while the phantom was included as a random effect. Similarly, all pre- and post-contrast $17 \mathrm{HB}$ MOLLI T1, $11 \mathrm{HB}$ MOLLI T1 as well as all ECV values quantified from volunteer data were also compared using a general linear mixed model. This model included time and the MOLLI protocols as fixed effects and the patients as random effect. The recovery rates of both the myocardial and 
blood pool post-contrast were compared using the Patrick Royston's p-trend test.

\section{Results}

\section{Phantom}

Both $11 \mathrm{HB}$ and $17 \mathrm{HB}$ MOLLI results were similar to IR-SE T1 values up to $\sim 500 \mathrm{~ms}$ but both sequences underestimated T1 time for values $>500 \mathrm{~ms}$. This underestimation was non linear and increased with higher heart rates. The $17 \mathrm{HB}$ MOLLI acquires 11 images whereas the $11 \mathrm{HB}$ MOLLI acquires 8 images. By comparing both sequences, the $11 \mathrm{HB}$ MOLLI has a predicted noise penalty of $17 \%$ compared to the $17 \mathrm{HB}$ MOLLI $(\sqrt{(11 / 8)})$. Comparing both sequences, the acquisition time was reduced by $35 \%$ when using the 11 HB MOLLI. Figure 1 and 2 respectively display the 17 $\mathrm{HB}$ and $11 \mathrm{HB}$ MOLLI results compared to IR-SE. There was no significant difference between the $11 \mathrm{HB}$ and 17 HB MOLLI $(p=0.82)$ by the general linear mixed model. A Bland-Altman plot comparing the two sequences at $60 \mathrm{bpm}$ is presented in Figure 3 and displays a mean difference of $0.45 \pm 1.96 \%$. There is no significant bias and no relationship between the magnitude of $\mathrm{T} 1$ and error.

\section{Volunteers}

All volunteers had normal renal function (eGFR: $108 \pm$ $17 \mathrm{ml} / \mathrm{min} / 1.73 \mathrm{~m}^{2}$ ) and no focal myocardial scars were detected on the LGE images. The pre- and post-contrast MOLLI images were all interpretable for T1 mapping and contained little to no artifacts. Figure 4 shows a mid-ventricular short axis T1 map for pre- and postcontrast. There was no significant difference between 11 $\mathrm{HB}$ and $17 \mathrm{HB}$ MOLLI in volunteers $(\mathrm{p}=0.41)$ by the general linear mixed model. All values reported in this study were therefore based on measurements acquired using the $11 \mathrm{HB}$ MOLLI only.

T1 values are field strength dependent and increase with field strength [15]. The mean pre-contrast T1 values of myocardium and blood were $1315 \pm 39 \mathrm{~ms}$

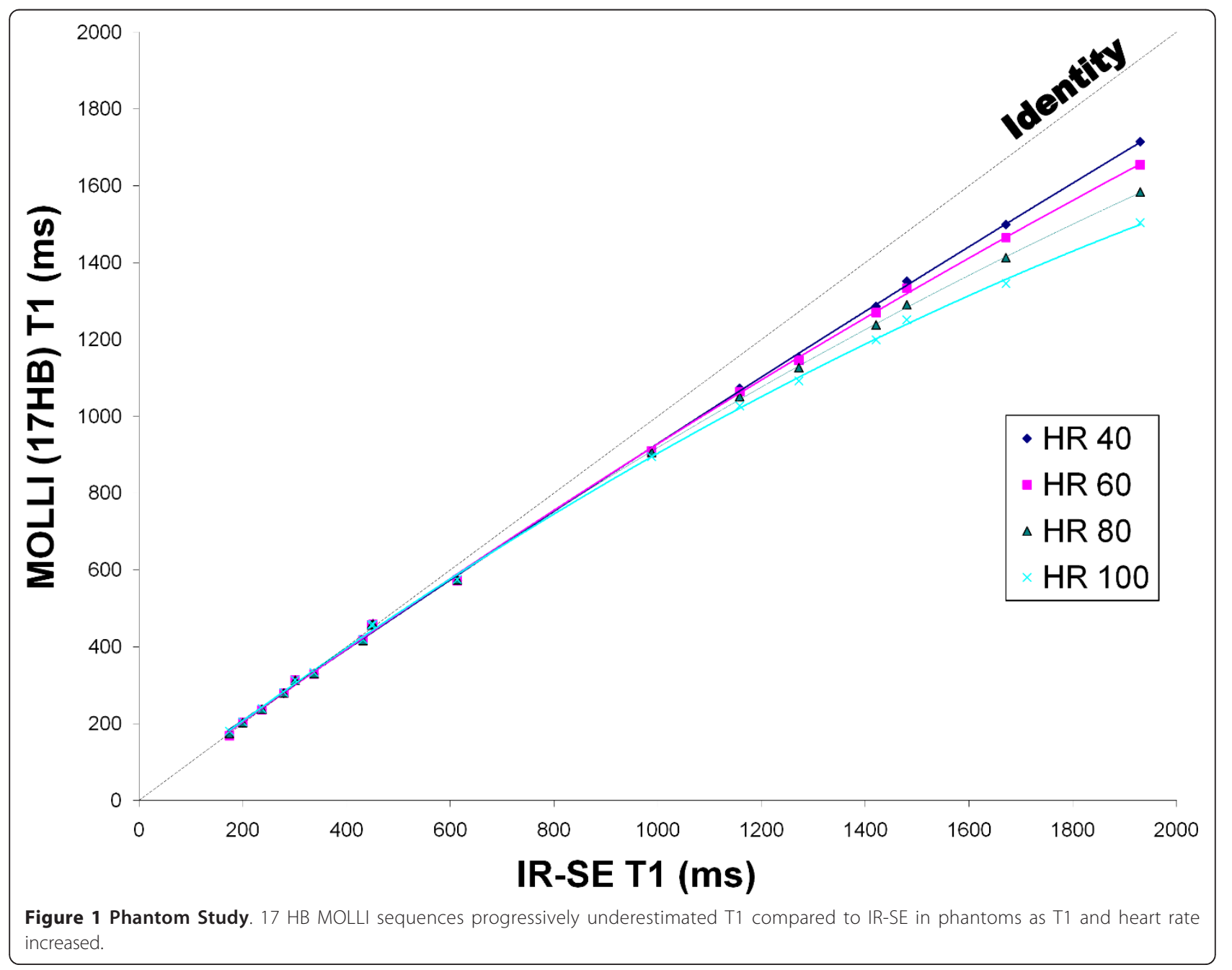




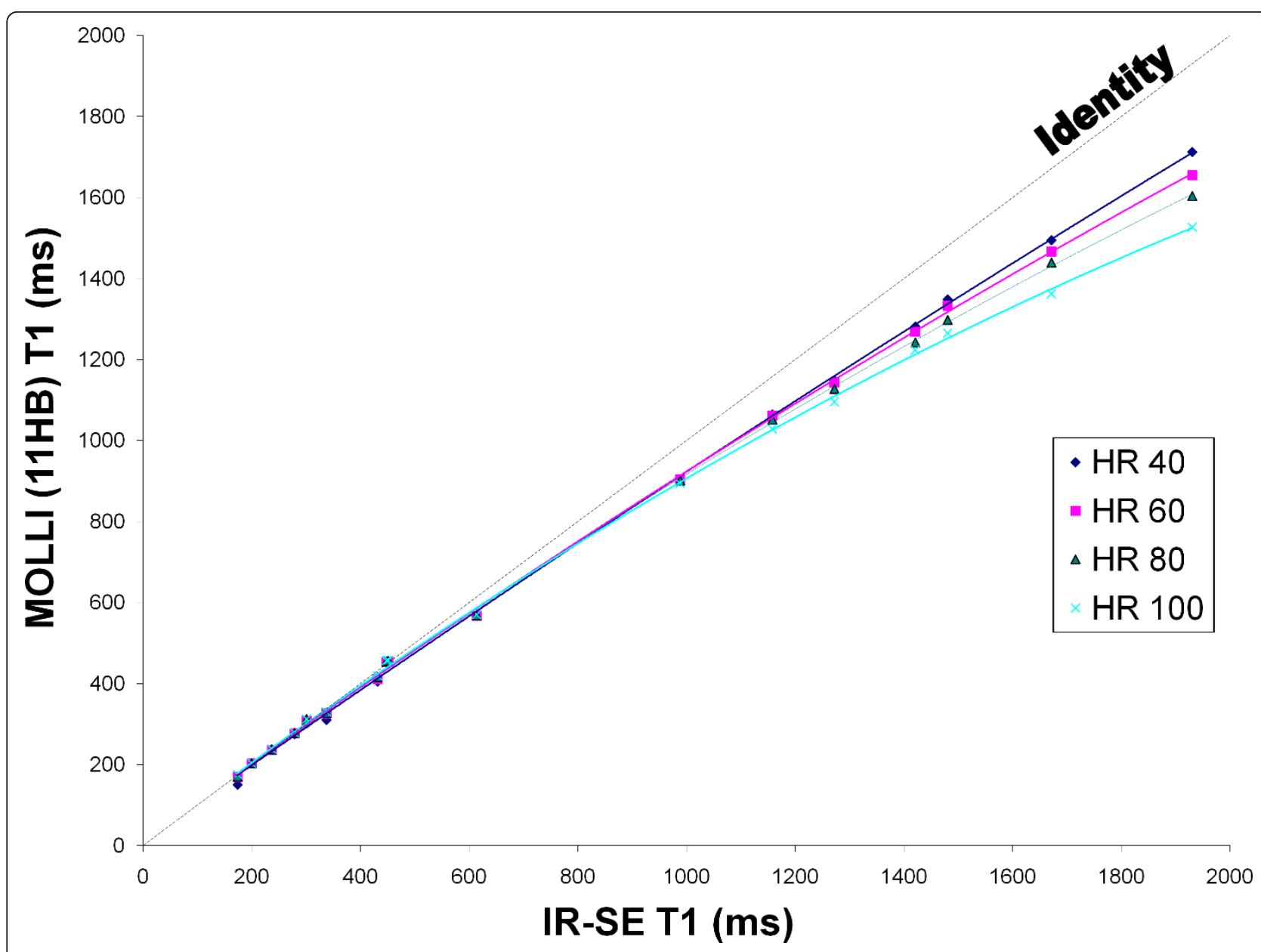

Figure 2 Phantom Study. 11 HB MOLLI sequences progressively underestimated T1 compared to IR-SE in phantoms as T1 and heart rate increased.

and $2020 \pm 129 \mathrm{~ms}$ respectively. A post-contrast myocardium and blood T1 plot is presented in Figure 5. This plot demonstrates that gadolinium contrast is at dynamic equilibrium between myocardium and blood pool where their respective $\mathrm{T} 1$ values are recovering at a similar rate from 3.5 to 23.5 minutes $(\mathrm{p}=0.86)$.

ECV was calculated at all post contrast time points by normalizing myocardial R1 change with blood R1 change and also correcting for hematocrit. Figure 6 displays the mean ECV values for each post-contrast time interval. There was no significant difference between 11 $\mathrm{HB}$ and $17 \mathrm{HB}$ ECV in volunteers $(\mathrm{p}=0.35)$. In addition, there is no significant difference of ECV after 8.5 minutes $(\mathrm{p}=0.10)$. The least square mean of ECV between 8.5 minutes and 23.5 minutes was $26.7 \pm 1.0 \%$. A Bland-Altman plot comparing the two sequences for all ECV values is presented in Figure 7.

Table 1 display myocardial and blood pool T1 values as well as myocardial ECV values using both the 17 $\mathrm{HB}$ and $11 \mathrm{HB}$ MOLLI sequences. The standard deviations (SD) for all T1 and ECV values are also presented.

\section{Discussion}

The MOLLI sequence has been demonstrated to be an accurate and reproducible approach for myocardial T1 mapping $[4,5]$. The originally described MOLLI protocol requires a 17 heartbeat breath hold. A breath hold duration of 17 heart beats is difficult to achieve for older subjects and patients with impaired cardiovascular or pulmonary function. In this study, we compared a two inversion-recovery block 11 heartbeat MOLLI with the classic three inversion-recovery block 17 heartbeat MOLLI. With a $35 \%$ reduction in acquisition time, the 11 HB MOLLI achieved excellent agreement with the 17 HB MOLLI over a wide range of T1 times in our phantom study. There was no statistical difference between the two protocols for normal subjects for both pre-contrast and a series of post-contrast time points at $3 \mathrm{~T}$. The $11 \mathrm{HB}$ MOLLI protocol is easy to implement on 


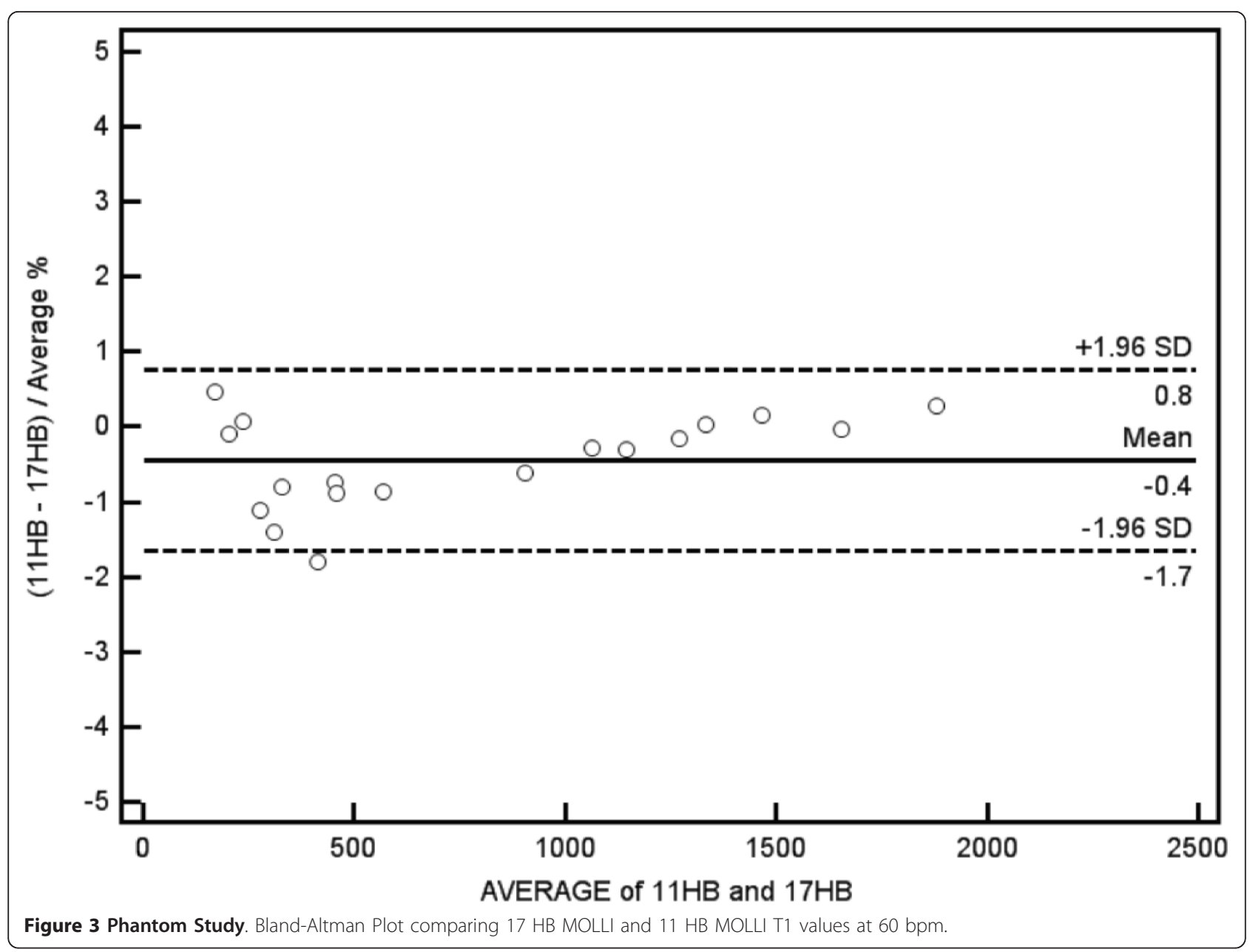

the scanner and requires no modification by post processing software. Both the $11 \mathrm{HB}$ MOLLI and $17 \mathrm{HB}$ MOLLI exhibited a systemic, nonlinear heart rate dependency of longer T1 values [5]. The observed deviation in measured versus actual T1 with lengthening T1 and shortened RR interval is as expected and does not display an inconsistent relationship. A heart rate correction of pre-contrast myocardial and blood $\mathrm{T} 1$ values is thereby recommended at 3 Tesla. Compared to shorter (and accurate) post-contrast T1's, the longer (and inaccurate) pre-contrast T1 times translated to R1 (1/ T1) have less effect on the ECV.

For T1 mapping to be a routine clinical tool, the 17 HB MOLLI protocol must have a significantly reduced acquisition time. Piechnik et al [6] proposed a 9 heartbeat shortened MOLLI (shMOLLI). Their sequence is a 3 inversion-recovery block scheme that collects 7 images in 9 heartbeats. Because of the insufficient magnetization recovery of the last 2 inversion-recovery blocks, the last 2 images were only used to fit the short T1 times

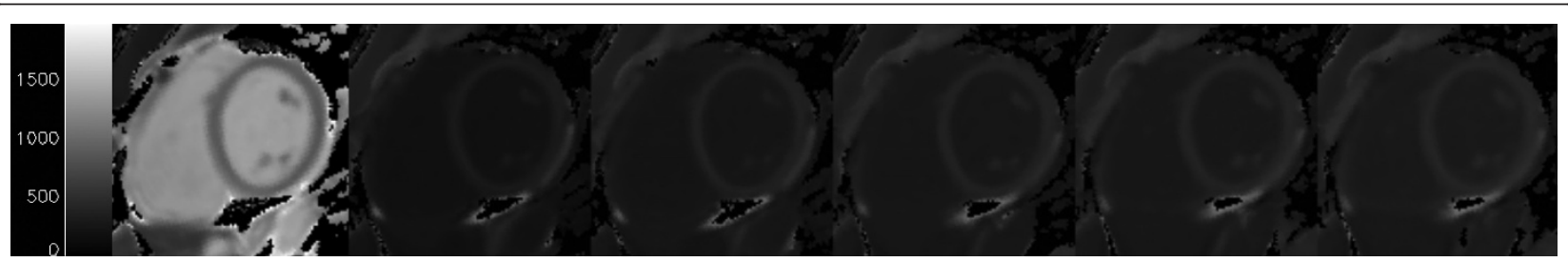

Figure 4 Human Study. Mid-ventricular short axis T1 maps. From left to right: pre-contrast, 5 minutes, 8.5 minutes, 13.5 minutes, 18.5 minutes and 23.5 minutes post-contrast. All images were displayed with same setting. 


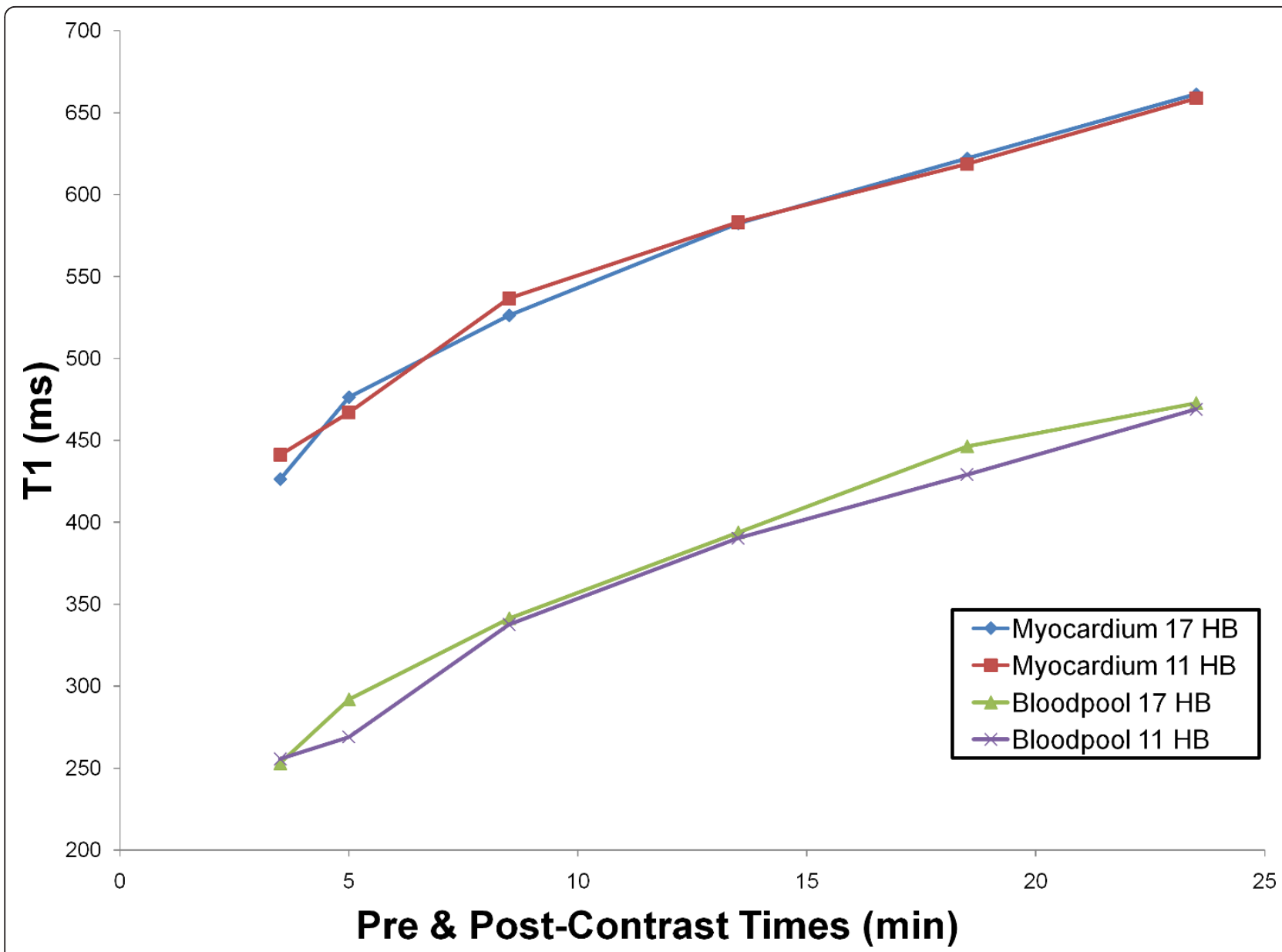

Figure 5 Human Study. Recovery of absolute myocardial and blood T1 from 3.5 minute to 23.5 minutes after 0.15 mmol/kg of Gd-DTPA.

by using a conditional fitting algorithm. The shMOLLI results displayed good correlation with $\mathrm{T} 1$ times less than $800 \mathrm{~ms}$, longer T1 times were underestimated about $4 \%$.

Imaging at $3 \mathrm{~T}$ has become the standard for neurological and musculoskeletal imaging. Cardiac imaging has been slow to adopt 3T imaging because of the inhomogeneities of both static magnetic field $\left(\mathrm{B}_{0}\right)$ and the transmit radiofrequency field $\left(B_{1}\right)$ [16]. With the recent technological development of parallel radiofrequency transmission [17] as well as improved shimming algorithms [18], 3T scanners have been a more viable option for cardiac imaging because of the improvements in signal-to-noise ratio.

Compared to limited literature reports, the pre-contrast blood T1 values measured in our study (2020 ms) showed similar mean values with the results presented in a study conducted by Stanisz et al [19] (1932 ms). In contrast, the results for both these studies are significantly longer than the results of studies conducted by Sharma et al [20] (1670 ms) and Noeske et al [16] (1550 $\mathrm{ms})$. This difference can be attributed to the limited range of TI values for these studies (100-800 ms and 500-1500 ms, respectively). The pre contrast blood T1 values from our data are about $26 \%$ to $38 \%$ longer than those of $1.5 \mathrm{~T}$ results. For pre-contrast myocardial T1 times, our results (1315 ms) are shorter than those obtained by Stanisz et al [19] (1471 ms), but longer than the results acquired by Piechnik et al [6] (1169 $\mathrm{ms})$, Sharma et al [20] (1200 ms) and Noeske et al [16] (1115 ms). Compared to previous myocardial T1 data at $1.5 \mathrm{~T}$, the pre-contrast myocardial $\mathrm{T} 1$ at $3 \mathrm{~T}$ are $22 \%$ to $33 \%$ greater.

Pre-contrast T1s are longer at 3T and may not fully recover when the same sampling schemes are used as for 1.5T. A study conducted by Schelbert et al [7] introduced a hybrid MOLLI protocol adapted to the expected pre-contrast or post-contrast $\mathrm{T} 1$ values. At $1.5 \mathrm{~T}$, longer pre-contrast T1 times used a 2 inversionrecovery block scheme that collects 6 images. The shorter post-contrast $\mathrm{T} 1$ times used a 3 inversion-recovery block scheme that collects 7 images to cover for 


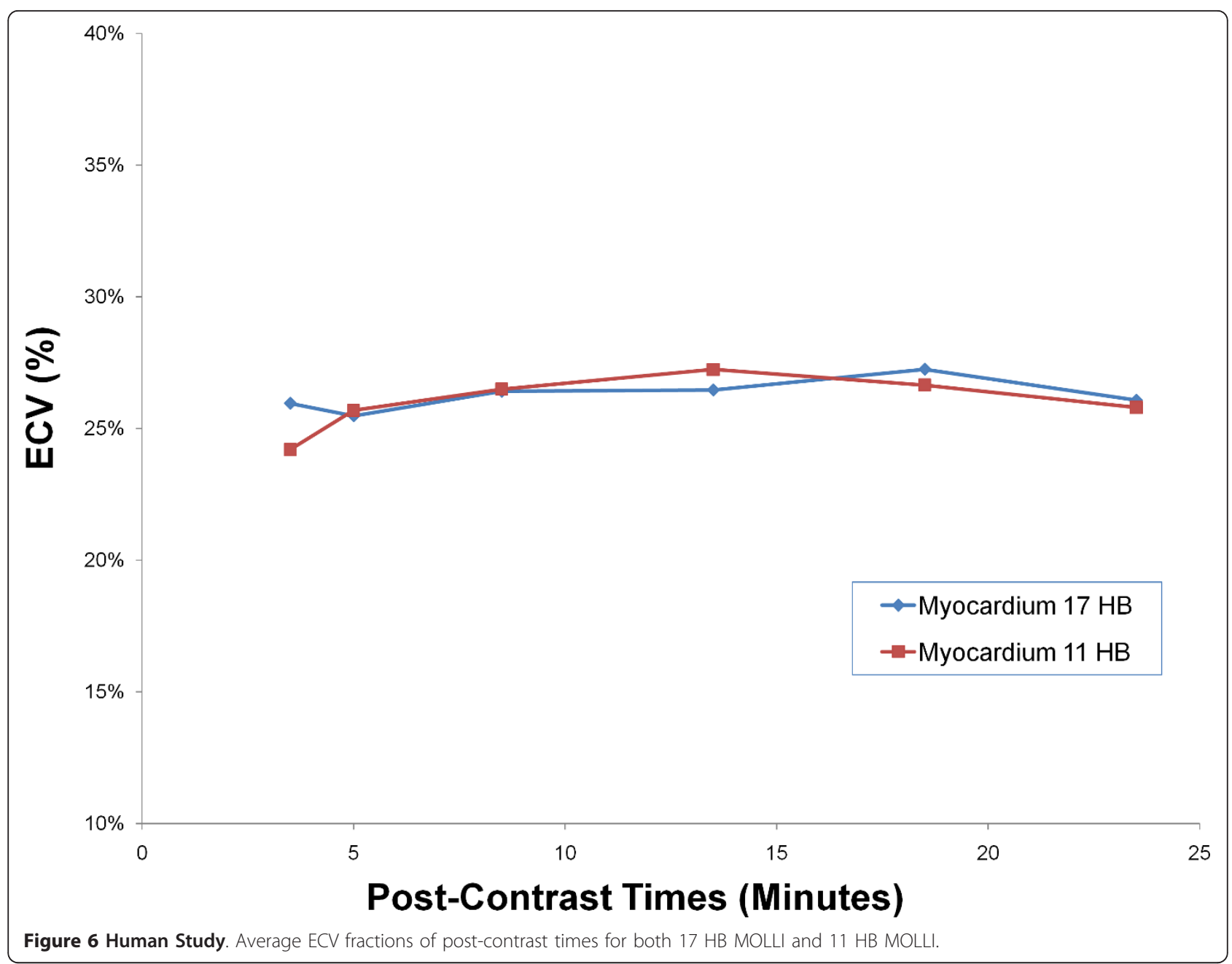

faster relaxation rates. Both the pre- and post-contrast scans were acquired in $11 \mathrm{HB}$. The hybrid MOLLI exhibits less bias and better agreement with the classic 17 HB MOLLI gold standard values at $1.5 \mathrm{~T}$. Breton et al [21] proposed a two heart beat saturation recovery $\mathrm{T} 1$ mapping technique. By acquiring a proton density image and a $500 \mathrm{~ms}$ saturation recovery image, T1 maps were generated by solving the Bloch equation. Although this method has very high acquisition speeds which effectively eliminate cardiac motion artifacts, it suffers from limited spatial resolution and limited T1 accuracy.

Extracellular volume fraction (ECV) [22,23], volume of distribution (Vd)[10], fibrosis index [24], and volume fraction of extravascular extracellular matrix (Ve) [7] all share the same parameters for measuring the myocardial extracellular matrix by adjusting the gadolinium contrast partition coefficient with the patient's hematocrit. A study conducted by Kehr et al [24] demonstrated that gadolinium distribution volume is closely correlated with histological collagen volume fraction (CVF) in vitro. Messroghli et al [12] recently reported there is a moderate correlation $(r=0.69)$ between CMR ECV and histological collagen volume fraction in a small animal model of left ventricular hypotrophy.

To accurately quantify ECV, steady state equilibrium of gadolinium chelates must be reached between the plasma and myocardial interstitium. Following an intravenous injection, gadolinium chelates such as Gd-DTPA are continuously cleared from the blood via renal clearance. After an initial period of equilibration, the contrast agent concentration in the blood will steadily decrease over time. However, if the contrast exchange rate between blood and tissue is faster than the renal clearance, then the ratio of contrast agent concentration between tissue and blood will, after the short initial equilibration period, achieve a dynamic equilibrium over a certain time [11], (Ugander et al, Extracellular volume imaging by MRI provides insight into overt and subclinical myocardial pathology, in press). Flett et al [10] developed the equilibrium contrast CMR method (EQCMR), by administering contrast via bolus injection followed by a slow continuous infusion. Their results 


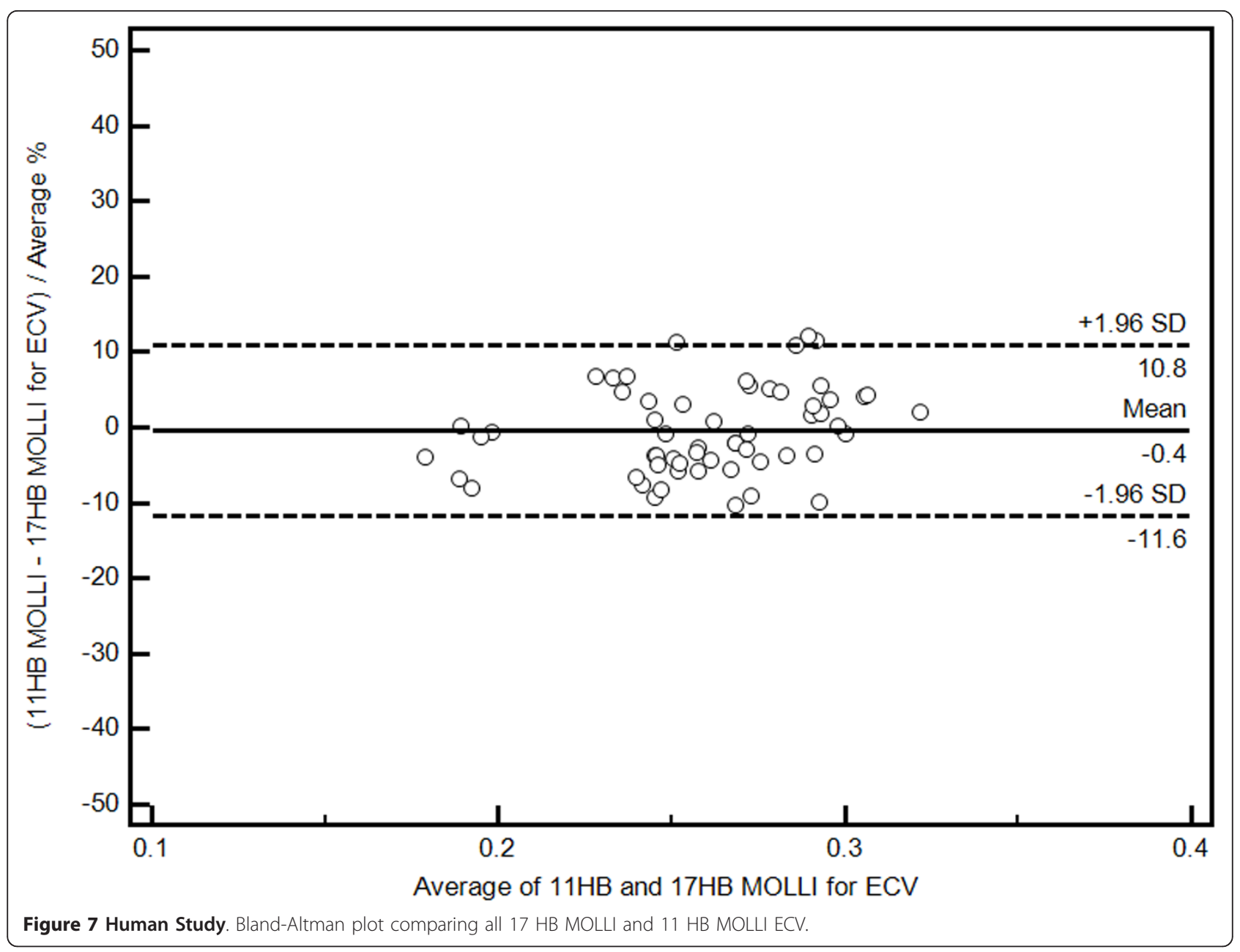

showed that volume of distribution correlates with histological CVF in aortic stenosis and hypertrophic cardiomypathy. However, longer infusion methods are difficult to incorporate into clinical CMR workflow. A previous study completed by Schelbert et al [7] validated the bolus technique as an equally accurate method as the slow infusion technique for quantifying ECV. We have verified this dynamic equilibrium in normal volunteer at $3 \mathrm{~T}$ by repeatedly measuring both myocardial and blood $\mathrm{T} 1$ at different time points. In certain pathological situations, such as the microvasular obstruction, the assumption of dynamic equilibrium is not valid.

Our study used the bolus injection technique. General linear mixed model demonstrated that there is no statistical difference of ECV values from 8.5 minutes. Between 8.5 to 23.5 minutes, the ECV differences between consecutive time points were less than $3 \%$. This difference is significantly less than the coefficient of variation for our average ECV value. Compared to previous reports, our results suggest that the gadolinium concentration could

Table 1 Myocardial and blood pool T1 as well as myocardial ECV values using both the $17 \mathrm{HB}$ and $11 \mathrm{HB}$ MOLLI sequences at different time points (Average \pm standard deviation)

\begin{tabular}{|c|c|c|c|c|c|c|c|c|}
\hline Structure & Sequence & Pre-Contrast & Post $3.5 \mathrm{~min}$ & Post $5 \mathrm{~min}$ & Post $8.5 \mathrm{~min}$ & Post $13.5 \mathrm{~min}$ & Post $18.5 \mathrm{~min}$ & Post $23.5 \mathrm{~min}$ \\
\hline Myocardium T1 (ms) & 17 HB MOLLI & $1,324 \pm 48$ & $426 \pm 44$ & $476 \pm 42$ & $526 \pm 31$ & $583 \pm 43$ & $622 \pm 40$ & $661 \pm 38$ \\
\hline Myocardium T1 (ms) & 11 HB MOLLI & $1,314 \pm 39$ & $441 \pm 50$ & $467 \pm 43$ & $537 \pm 52$ & $583 \pm 48$ & $619 \pm 48$ & $659 \pm 52$ \\
\hline Blood pool T1 (ms) & 17 HB MOLLI & $2,037 \pm 152$ & $246 \pm 33$ & $282 \pm 38$ & $331 \pm 37$ & $384 \pm 38$ & $433 \pm 42$ & $462 \pm 41$ \\
\hline Blood pool T1(ms) & 11 HB MOLLI & $2,020 \pm 128$ & $255 \pm 39$ & $269 \pm 22$ & $337 \pm 43$ & $390 \pm 44$ & $429 \pm 39$ & $469 \pm 42$ \\
\hline Myocardium ECV (\%) & 17 HB MOLLI & N/A & $25.5 \pm 4.1$ & $26.4 \pm 3.8$ & $26.5 \pm 3.8$ & $27.3 \pm 4.2$ & $26.1 \pm 3.4$ & $26.3 \pm 3.8$ \\
\hline Myocardium ECV (\%) & 11 HB MOLLI & N/A & $24.2 \pm 3.8$ & $25.7 \pm 3.8$ & $26.5 \pm 4.4$ & $27.2 \pm 4.6$ & $26.7 \pm 3.9$ & $25.8 \pm 4.1$ \\
\hline
\end{tabular}


reach dynamic equilibrium between plasma and myocardium as early as 8.5 minutes. Combining our results with those of Schelbert et al [7], ECV is stable in normal volunteers from 8.5 to 50 minutes after bolus contrast injection. Our findings permit considerable flexibility for incorporating ECV into routine CMR work flow. A limitation of both this study and Schelbert's study is that there is a lack of substantial data for subjects with cardiac disease. Diseased subjects may have areas of severe fibrosis/scarring or cardiac dysfunction. Either of these conditions could delay the time to reach steady-state equilibrium. Further testing in patients with severe fibrosis and reduced ejection fraction should be done to verify whether continuous infusions can be fully replaced by the single-bolus approach in clinical settings.

$\mathrm{T} 1$ values are affected by confounding variables such as field strength, gadolinium contrast type and dose, scanning time and the patient's renal function. Due to these factors, $\mathrm{T} 1$ times cannot be readily compared to $\mathrm{T} 1$ data from other centers. In contrast, ECV is an inherent physiological property that should not be affected by these variables. The ECV data of normal volunteers from our study at 3T (26.7\%) slightly higher than the "fibrosis index" of normal volunteers reported by Broberg et al [9] (24.8\%) and the Ve quantified by Schelbert et al [7] after the Ve value was converted into comparable ECV values (24.1\%). The higher ECV seen in this study might be an effect of relatively stronger shortening of the longer pre-contrast blood $\mathrm{T} 1$ at $3 \mathrm{~T}$ as compared to that at $1.5 \mathrm{~T}$. In addition, Both Broberg and Schelbert's study used protocols significantly different from the protocol used in our study.

\section{Conclusions}

In conclusion, we have validated MOLLI sequences at $3 \mathrm{~T}$ in phantom studies. We present values for myocardial and blood T1 pre and post gadolinium contrast at 3T. At 3T, post-gadolinium ECV is stable between 8.5 and 23.5 minutes after gadolinium injection.

\section{Limitations}

There are several limitations to this study. This studied had a small number of subjects with varying ages and genders. Only a single contrast agent was used for this study. There is no direct comparison to EQCMR or ShMOLLI. There is no repeatability analysis with intra and inter-observer variability. Only the mid-cavity ventricular slice was taken and the mean T1 for the entire myocardium was used. There may be some variations in T1 and ECV in the basal, apical, septal and lateral walls.

\section{Acknowledgements}

Research funding was provided by the NIH intramural research program. We thank Jacquin L. Jones, RN for recruiting the volunteers.

\section{Author details}

'Radiology and Imaging Sciences, National Institutes of Health Clinical Center, Bethesda, MD, USA. ${ }^{2}$ Molecular Biomedical Imaging Laboratory, National Institute of Biomedical Imaging and Bioengineering, Bethesda, MD, USA. ${ }^{3}$ Laboratory of Cardiac Energetics, National Heart, Lung and Blood Institute, Bethesda, MD, USA. ${ }^{4}$ U.S. Food and Drug Administration, Rockville, $M D$, USA.

\section{Authors' contributions}

All authors read, critically edited the initial manuscript, added intellectual content, and approved the final version. DAB and SL designed, coordinated and conducted the study, MU created the phantoms. SL and MSN acquired all data, JJL and NK analyzed all data, JH conducted the statistical analyses. PK assisted with pulse sequence optimization. AEA assisted with the study design, data analysis, and added critical manuscript content.

\section{Competing interests}

The authors declare that they have no competing interests.

Received: 14 June 2011 Accepted: 28 November 2011

Published: 28 November 2011

\section{References}

1. Hundley WG, Bluemke DA, Finn JP, Flamm SD, Fogel MA, Friedrich MG, Ho VB, Jerosch-Herold M, Kramer CM, Manning WJ, et al: ACCF/ACR/AHA/ NASCI/SCMR 2010 expert consensus document on cardiovascular magnetic resonance: a report of the American College of Cardiology Foundation Task Force on Expert Consensus Documents. J Am Coll Cardiol 2010, 55:2614-2662.

2. Marijianowski MM, Teeling P, Mann J, Becker AE: Dilated cardiomyopathy is associated with an increase in the type I/type III collagen ratio: a quantitative assessment. J Am Coll Cardiol 1995, 25:1263-1272.

3. Vogel-Claussen J, Rochitte CE, Wu KC, Kamel IR, Foo TK, Lima JA, Bluemke DA: Delayed enhancement MR imaging: utility in myocardial assessment. Radiographics 2006, 26:795-810.

4. Messroghli DR, Greiser A, Frohlich M, Dietz R, Schulz-Menger J: Optimization and validation of a fully-integrated pulse sequence for modified look-locker inversion-recovery (MOLLI) T1 mapping of the heart. J Magn Reson Imaging 2007, 26:1081-1086.

5. Messroghli DR, Plein S, Higgins DM, Walters K, Jones TR, Ridgway JP, Sivananthan MU: Human myocardium: single-breath-hold MR T1 mapping with high spatial resolution-reproducibility study. Radiology 2006, 238:1004-1012.

6. Piechnik SK, Ferreira VM, Dall'Armellina E, Cochlin LE, Greiser A, Neubauer S, Robson MD: Shortened Modified Look-Locker Inversion recovery (ShMOLLI) for clinical myocardial T1-mapping at 1.5 and $3 \mathrm{~T}$ within a 9 heartbeat breathhold. J Cardiovasc Magn Reson 2010, $12: 69$.

7. Schelbert EB, Testa SM, Meier CG, Ceyrolles WJ, Levenson JE, Blair AJ, Kellman P, Jones BL, Ludwig DR, Schwartzman D, et al: Myocardial extravascular extracellular volume fraction measurement by gadolinium cardiovascular magnetic resonance in humans: slow infusion versus bolus. J Cardiovasc Magn Reson 2011, 13:16.

8. Gai N, Turkbey EB, Nazarian S, Van Der Geest RJ, Liu CY, Lima JA, Bluemke DA: T1 mapping of the gadolinium-enhanced myocardium: adjustment for factors affecting interpatient comparison. Magnetic resonance in medicine: official journal of the Society of Magnetic Resonance in Medicine/Society of Magnetic Resonance in Medicine 2011, 65:1407-1415.

9. Broberg CS, Chugh SS, Conklin C, Sahn DJ, Jerosch-Herold M: Quantification of diffuse myocardial fibrosis and its association with myocardial dysfunction in congenital heart disease. Circ Cardiovasc Imaging 2010, 3:727-734.

10. Flett AS, Hayward MP, Ashworth MT, Hansen MS, Taylor AM, Elliott PM, Mcgregor C, Moon JC: Equilibrium Contrast Cardiovascular Magnetic Resonance for the Measurement of Diffuse Myocardial Fibrosis. Preliminary Validation in Humans. Circulation 2010, 122:138-144.

11. Arheden $H$, Saeed M, Higgins CB, Gao DW, Bremerich J, Wyttenbach R, Dae MW, Wendland MF: Measurement of the distribution volume of gadopentetate dimeglumine at echo-planar MR imaging to quantify myocardial infarction: comparison with 99mTc-DTPA autoradiography in rats. Radiology 1999, 211:698-708. 
12. Messroghli D, Nordmeyer S, Dietrich T, Dirsch O, Kaschina E, Savvatis K, D OHI, Klein C, Berger F, Kuehne T: Assessment of Diffuse Myocardial Fibrosis in Rats Using Small Animal Look-Locker Inversion Recovery (SALLI) T1 Mapping. Circulation Cardiovascular imaging 2011, 4:636-640.

13. Kellman P, Arai AE, Mcveigh ER, Aletras AH: Phase-sensitive inversion recovery for detecting myocardial infarction using gadolinium-delayed hyperenhancement. Magn Reson Med 2002, 47:372-383.

14. Messroghli DR, Rudolph A, Abdel-Aty H, Wassmuth R, Kuhne T, Dietz R, Schulz-Menger J: An open-source software tool for the generation of relaxation time maps in magnetic resonance imaging. BMC Med Imaging 2010, 10:16.

15. Bottomley PA, Foster TH, Argersinger RE, Pfeifer LM: A review of normal tissue hydrogen NMR relaxation times and relaxation mechanisms from 1-100 MHz: dependence on tissue type, NMR frequency, temperature, species, excision, and age. Med Phys 1984, 11:425-448.

16. Noeske $R$, Seifert $F$, Rhein $\mathrm{KH}$, Rinneberg $\mathrm{H}$ : Human cardiac imaging at $3 \mathrm{~T}$ using phased array coils. Magn Reson Med 2000, 44:978-982.

17. Willinek WA, Gieseke J, Kukuk GM, Nelles M, Konig R, Morakkabati-Spitz N, Traber F, Thomas D, Kuhl CK, Schild HH: Dual-source parallel radiofrequency excitation body MR imaging compared with standard MR imaging at 3.0 T: initial clinical experience. Radiology 2010, 256:966-975.

18. Schar M, Vonken EJ, Stuber M: Simultaneous B(0)- and B(1)+-map acquisition for fast localized shim, frequency, and RF power determination in the heart at 3 T. Magn Reson Med 2010, 63:419-426.

19. Stanisz GJ, Odrobina EE, Pun J, Escaravage M, Graham SJ, Bronskill MJ, Henkelman RM: T1, T2 relaxation and magnetization transfer in tissue at 3T. Magn Reson Med 2005, 54:507-512.

20. Sharma P, Socolow J, Patel S, Pettigrew Rl, Oshinski JN: Effect of Gd-DTPABMA on blood and myocardial T1 at $1.5 \mathrm{~T}$ and $3 \mathrm{~T}$ in humans. J Magn Reson Imaging 2006, 23:323-330.

21. Breton E, Kim D, Chung S, Axel L: Rapid Cardiac T1 Mapping within Two Heartbeats. Journal of Cardiovascular Magnetic Resonance 2011, 13:0107.

22. Kroll H, Korman S, Siegel E, Hart HE, Rosoff B, Spencer H, Laszlo D: Excretion of yttrium and lanthanum chelates of cyclohexane 1,2-trans diamine tetraacetic acid and diethylenetriamine pentaacetic acid in man. Nature 1957, 180:919-920

23. Kruhoffer P: Inulin as an Indicator for the Extracellular Space. Acta Physiologica Scandinavica 1946, 11:16-36.

24. Kehr E, Sono M, Chugh SS, Jerosch-Herold M: Gadolinium-enhanced magnetic resonance imaging for detection and quantification of fibrosis in human myocardium in vitro. Int J Cardiovasc Imaging 2008, 24:61-68.

doi:10.1186/1532-429X-13-75

Cite this article as: Lee et al: Myocardial $\mathrm{T} 1$ and Extracellular Volume

Fraction Mapping at 3 Tesla. Journal of Cardiovascular Magnetic Resonance 2011 13:75.

\section{Submit your next manuscript to BioMed Central and take full advantage of:}

- Convenient online submission

- Thorough peer review

- No space constraints or color figure charges

- Immediate publication on acceptance

- Inclusion in PubMed, CAS, Scopus and Google Scholar

- Research which is freely available for redistribution

Submit your manuscript at www.biomedcentral.com/submit 